year a total of $253(24.5 \%)$ patients had died. The KaplanMeier curves in figure 1 demonstrate a positive association between mortality and increasing hs-cTnI concentrations relative to the ULN. Specifically, using the log-rank test, the mortality at one year was significantly higher $(p<0.001)$ in patients with hs-cTnI concentrations above the ULN. Furthermore, on multivariable Cox regression analysis, the $\log (10)$ hscTnI concentration was independently associated with the hazard of one year mortality (hazard ratio 1.587 (95\% confidence interval $1.358-1.856$ ).

Conclusions These data suggest that admission hs-cTnI is a biomarker for one year mortality in critical care patients. Further work is now required to assess whether any medical intervention can alter this risk.

Conflict of Interest All of these assays used in our studies were provided by Beckman Coulter but they had no other role in the studies

\section{RETROSPECTIVE ANALYSIS OF THE IMPACT OF FULL VS CULPRIT ONLY REVASCULARISATION IN OCTOGENARIANS WITH MULTI-VESSEL CORONARY ARTERY DISEASE}

Nicolas Buttinger, Jonathan Smith, Adam de Belder. Brighton and Sussex University Hospitals NHS Trust, Brighton, UK

\subsection{6/heartjnl-2021-BCS.47}

Objectives The aim of this study was to evaluate all-cause mortality in patients aged greater than 80 years old with multi-vessel (MV) coronary artery disease (CAD) presenting with an acute ST segment elevation myocardial infarction (STEMI) and the impact of culprit only (CORV) versus full revascularisation (FRV).

Background Current European Society of Cardiology (ESC) guidelines recommend FRV either during the index procedure or as a staged approach in patients presenting with STEMIs and found to have MV CAD. A recent meta-analysis demonstrated a 31\% relative risk reduction in cardiovascular mortality in patients undergoing FRV. The average age of patients in the trials that have directed these guidelines is 61-65 and therefore their applicability to the elderly is questionable.

Methods This is a retrospective cohort study of patients presenting with a STEMI to the Royal Sussex County Hospital between January 2009 and December 2019. Groups were defined as those less than or greater than 80 years old and subdivided into those with single vessel (SV) or MV CAD. Group baseline characteristics were compared and the time to all-cause mortality from index procedure was assessed for each group. Logrank tests / cox-regression models were used for survival analysis and chi-squared tests for categorical data. Significance level $=<0.05$.

Results 2809 eligible patients were identified during the study period. In those less than 80 years old (2418 patients) 1751 patients had SV CAD and 647 (26.8\%) had MV CAD. Of those with MV CAD, 81 (12.5\%) underwent FRV. In patients greater than 80 years old (391 patients) 247 patients had SV CAD and 144 (36.8\%) had MV CAD of which $19(13.2 \%)$ had FRV. Patients greater than 80 years old were significantly more likely to have MV CAD compared with patients less than 80 years old $(p<0.0001)$. There was no significant difference between the two groups in the number of patients with MV CAD undergoing FRV $(p=0.78)$. Patients less than 80 with MV CAD had a significantly higher mortality rate than those with SV CAD (HR 1.47 [95\% CI 1.13-1.91], p=0.004). (Figure 1B). Whereas in patients greater than 80 years old

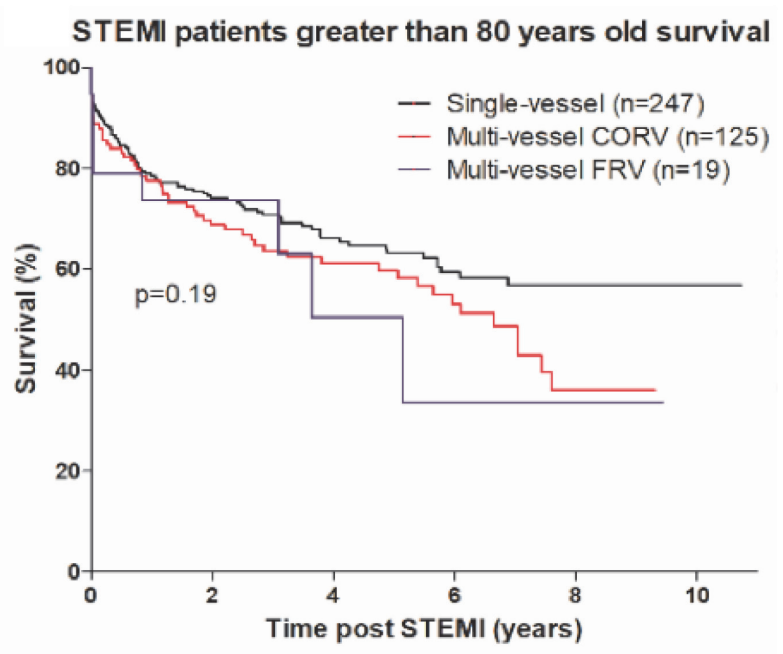

Abstract 47 Figure 2 There was no difference in mortality between patients $>80$ years old with MV CAD who underwent FRV or CORV $(p=0.19)$
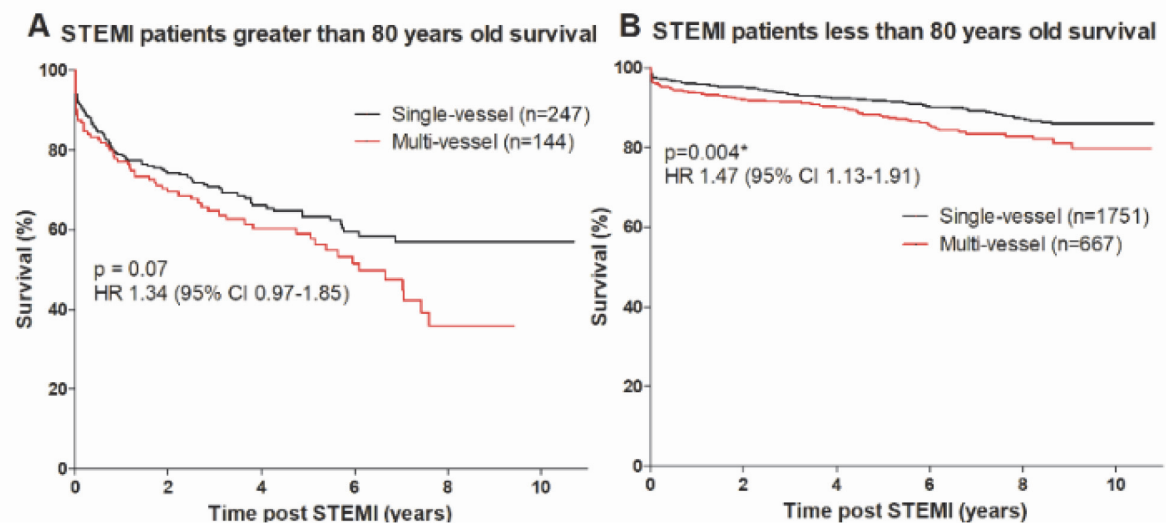

Abstract 47 Figure $1 \mathrm{~A}$ : There was no significant difference in mortality between patients with and without MVCAD in patients $>80$ years old $(p=0.07)$. B: There was significantly higher mortality rate in patients $<80$ with MVCAD compared to those with single vessel disease (HR 1.47 (1.131.91), $\mathrm{p}=0.004)$ 
there was no significant difference in mortality rates between those with and without MV CAD (HR 1.34 [95\% CI 0.971.85], $\mathrm{p}=0.07)$. (Figure 1A). There was also no significant difference in mortality in patients with MV CAD greater than 80 years old undergoing CORV versus FRV $(p=0.19)$. (Figure 2).

Conclusions The results show there is a mortality difference in patients with MV versus SV CAD in patients less than 80 years old, which is not seen in patients greater than 80 years old. There also does not appear to be a mortality benefit in FRV compared with CORV in the elderly. While a reduction in cardiovascular mortality with FRV has been demonstrated in a recent meta-analysis these results indicate that this benefit may be tempered in elderly patients. This is a single centre study with relatively small numbers, especially in those greater than 80 years old undergoing FRV. Given the uncertainty around FRV in the elderly, there remains a need for a randomised control trial to evaluate this question.

Conflict of Interest None

\section{DRUG COATED BALLOON ONLY ANGIOPLASTY FOR STEMI}

${ }^{1}$ Ioannis Merinopoulos, ${ }^{2}$ Tharusha Gunawardena, ${ }^{2}$ Natasha Corballis, ${ }^{3}$ Upul Wickramarachchi, ${ }^{3}$ Clint Maart, ${ }^{3}$ Sulfi Sreekumar, ${ }^{3}$ Chris Sawh, ${ }^{3}$ Trevor Wistow, ${ }^{3}$ Toomas Sarev, ${ }^{3}$ Tim Gilbert, ${ }^{3}$ Alisdair Ryding, ${ }^{4}$ Vassilios Vassiliou, ${ }^{3}$ Simon Eccleshall. ${ }^{1}$ University of East Anglia, Norfolk \& Norwich University Hospital, Norwich, UK; ${ }^{2}$ Norwich Medical School and Norfolk \& Norwich University Hospital; ${ }^{3}$ Norfolk \& Norwich University Hospital; ${ }^{4}$ Norwich Medical School, University of East Anglia

\subsection{6/heartjnl-2021-BCS.48}

Introduction In the setting of primary percutaneous coronary intervention (PPCI), it has been shown that stenting compared to balloon angioplasty (BA) reduces the need for repeat revascularisation but not overall mortality. A recent, small randomised trial showed that drug coated balloon (DCB) angioplasty for PPCI was non-inferior to drug eluting stent (DES) in terms of fractional flow reserve at 9 months. Our aim was to investigate the safety of DCB angioplasty in a STEMI population in terms of all-cause mortality.

Methods We identified all patients treated for STEMI in our institution from January 2016 until November 2019. We excluded patients who died in hospital and patients with cardiac arrest, cardiogenic shock or requiring intubation; given that their mortality is more likely to be determined by the severity of their clinical presentation rather than the PCI strategy. The primary endpoint was all-cause mortality. Survival data were obtained through the UK Health and Social Care Information Service. Clinical and angiographic data were collected from our prospectively collated database and supplemented from electronic records where required. All angiograms were reviewed by an experienced operator to confirm treatment strategy, bifurcation lesions, coronary artery dissection and TIMI flow pre- and post-intervention.

Results A total of 1190 patients were identified; 470 treated with DCB and 720 with DES. The average age for the DCB group was $65.5 \pm 12.5$ years old $(73.6 \%$ male); while for the DES group it was $65.5 \pm 11.6$ years old $(74.7 \%$ males). The average follow up was $882 \pm 439$ days and $978 \pm 426$ days for the DCB and DES group respectively. There were a few differences between the groups (table 1).
Abstract 48 Table 1

\begin{tabular}{|l|l|l|l|}
\hline & DCB (470) & DES (720) & P value \\
\hline HTN & $192(40.9 \%)$ & $251(34.9 \%)$ & 0.037 \\
\hline Smoking & $264(56.2 \%)$ & $455(63.2 \%)$ & 0.006 \\
\hline Bifurcations & $196(41.7 \%)$ & $213(29.6 \%)$ & $<0.001$ \\
\hline True bifurcations & $49(10.4 \%)$ & $40(5.6 \%)$ & 0.002 \\
\hline Vessel diameter & $3.28(0.54)$ & $3.52(0.6)$ & $<0.001$ \\
\hline
\end{tabular}

Abstract 48 Table 2

\begin{tabular}{|l|l|l|}
\hline & Hazard ratio (95\% CI) & P value \\
\hline Age & $1.081(1.058,1.106)$ & $<0.001$ \\
\hline Stroke & $2.453(1.148,5.243)$ & 0.021 \\
\hline Coronary artery bypass graft & $4.776(1.863,12.241)$ & 0.001 \\
\hline Heart failure & $5.121(1.314,19.964)$ & 0.019 \\
\hline COPD & $2.489(1.266,4.895)$ & 0.008 \\
\hline Diabetes & $1.831(1.063,3.153)$ & 0.029 \\
\hline
\end{tabular}

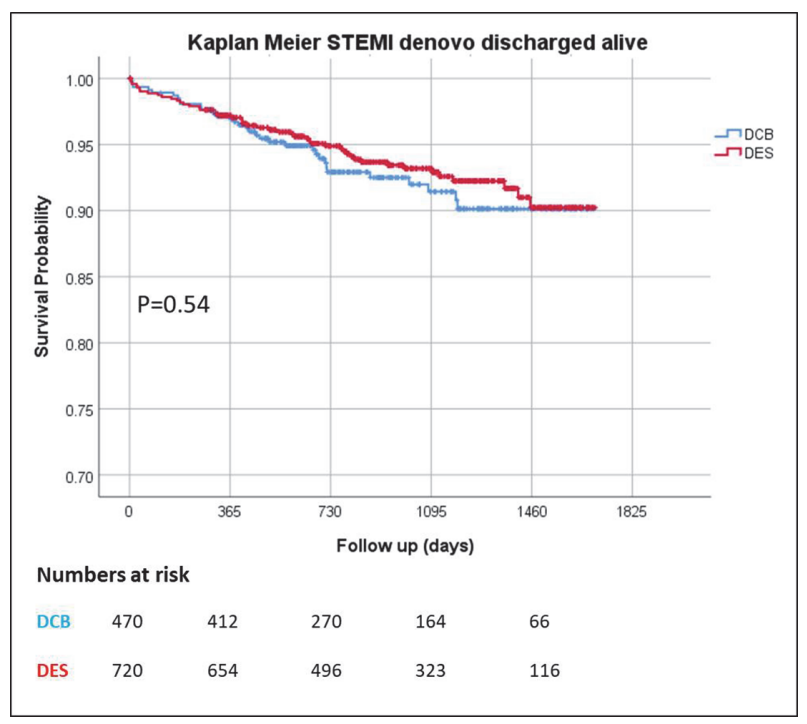

Abstract 48 Figure 1

There were no differences in all other clinical and angiographic characteristics. The all-cause mortality was $33 / 470$ (7\%) for the DCB group and 48/720 (6.7\%) for the DES group. Kaplan Meier estimator plot for all-cause mortality (figure 1) did not show a significant difference between DCB and DES $(p=0.54)$. On multivariable Cox regression analysis (table 2), age, prior stroke, coronary artery bypass graft, heart failure, chronic obstructive pulmonary disease (COPD) and diabetes were significant poor predictors of mortality.

Conclusion Our real-world data from a large, contemporary cohort of STEMI patients demonstrate that DCB only angioplasty is safe compared to DES and may be considered as a treatment option.

Conflict of Interest none 